www.jmscr.igmpublication.org

Impact Factor (SJIF): 6.379

Index Copernicus Value: 79.54

ISSN (e)-2347-176x ISSN (p) 2455-0450

crossrefDOI: https://dx.doi.org/10.18535/jmscr/v7i1.47

\title{
Evaluation of Gestational age by Sonological Measurement of Placental Thickness
}

\author{
Author \\ Dr Himanshu Kumar Jaiswal \\ Junior Resident, Department of Radiology, Katihar Medical College
}

\section{Introduction}

The role of sonography in the evaluation of morphology and detection of placental abnormalities in clinical conditions such as nonimmune hydrops, gestational diabetes, chorioangioma, intraplacental hemorrhage and intrauterine growth restriction has been well established.

Placenta is primarily a fetal organ and its size is a reflection of the health and size of the fetus. One additional ultrasonographic parameter frequently used to assess the placenta is placental size. ${ }^{[1]}$

The measurement of placental thickness is relatively simple and clinically useful. Abnormal thickness of placenta is well recognized as a diagnostic harbinger in a wide spectrum of pathologic events. Placental thickness can contribute to the management of fetus at risk. ${ }^{[2]}$ Virtually, all the important clinical decisions, which include caesarean section, elective labour induction, etc., depend on the knowledge of the gestational age and placental position.

The role of placental thickness as a new parameter for estimating gestational age and placental thickness normograms in relation to gestational age have been published. Placental thickness measurement can differentiate normal from abnormal pregnancy. ${ }^{[3]}$

Ultrasonography (USG) is commonly used to estimate the gestational age by measuring the fetal dimensions like the Biparietal Diameter (BPD), the Abdominal Circumference (AC), the Head Circumference (HC) and the Femur Length (FL).

There are some drawbacks in those above said parameters in estimating the gestational age. So, there is a need of another parameter for supplementing the gestational age estimation with minimal error. Nyberg and Finberg reported that the placental thickness parallels the gestational age ${ }^{[4]}$.

In our present study we have tried to measure placental thickness at the level of umbilical cord and determine its relationship with gestational age of fetus in normal singleton pregnancy.

\section{Aim}

- To evaluate placental thickness, measured at the insertion of the umbilical cord, as a parameter for estimating gestational age of the fetus in singleton pregnancies. 


\section{Materials and Methods}

We studied 500 antenatal cases of gestational age more than 10 weeks of gestation based on Crown Rump Length and gestational sac diameter up to $11^{\text {th }}$ week and thereafter by measuring BPD, HC, AC and FL from August 2017 - October 2018.

\section{Results}

The placental thickness gradually increased from $11.36 \mathrm{~mm}$ at 11 weeks to $36.50 \mathrm{~mm}$ at 40 weeks.

From 11 to 35 weeks of gestation, placental thickness in millimeter almost matched the gestational age in weeks. Thereafter, from $36-40$ weeks, placental thickness was less than gestational age by $1-3 \mathrm{~mm}$. At no stage of pregnancy was the normal placenta greater than $38 \mathrm{~mm}$.

Placental thickness increases with gestational age. It is observed that the relationship of placental thickness with the gestational age falls marginally and the rate of growth of placental thickness decreases after 36 weeks of gestation.

For every week of increase in gestational age there is an average increase of placental thickness by $0.8901 \mathrm{~mm}$.
Gestational Age (weeks) Gestational Age (weeks)

Number of subjects Number of subjects

$\%$ of subjects

\%of subjects

2.2
1.2
4.4
4.0
3.2
2.8
3.6
2.0
4.0
5.2
4.6
4.8
4.0
2.4
4.0

2.2

1.2

4.4

4.0

3.2

2.8

3.6

2.0

4.0

5.2

4.6

4.8

4.0

2.4

4.0

$\%$ of subjects

PT (mm) Mean
\pm SD

3.2

3.6

3.0

2.4

2.8

2.2

3.2

4.6

3.4
PT $(\mathbf{m m})$ Mean \pm SD

PT $(\mathbf{m m})$ Mean \pm SD

$11.36 \pm 0.50$
$12.00 \pm 0.00$
$14.27 \pm 2.88$
$14.00 \pm 0.73$
$15.75 \pm 1.06$
$16.07 \pm 1.21$
$17.06 \pm 0.24$
$17.70 \pm 1.06$
$19.45 \pm 1.19$
$20.73 \pm 1.28$
$21.00 \pm 1.35$
$22.58 \pm 1.28$
$23.30 \pm 1.13$
$24.83 \pm 0.94$
$24.80 \pm 1.36$

$26.63 \pm 1.45$

$27.28 \pm 1.71$

$28.47 \pm 0.92$

$28.67 \pm 1.44$

$28.57 \pm 3.63$

$30.55 \pm 1.44$

$31.19 \pm 1.87$

$31.91 \pm 1.04$

$34.41 \pm 1.12$
CI95\% CI 95\%

CI CIower- uppeCIr)
$11.02-11.70$
12.00-12.00
12.99-15.55
$13.7-14.3$
$15.2-16.3$
$15.4-16.8$
$16.9-17.2$
$16.9-18.5$
$18.9-20$
$20.2-21.2$
20.4 - 21.6
$22-23.1$
$22.8-23.8$
$24.2-25.4$
$24.2-25.4$

95\% CI (lowerupper)

$25.8-27.4$

26.4 - 28.1

$28-29$

27.8 - 29.6

$26.5-30.7$

29.6 - 31.5

$30.2-32.2$

31.5 - 32.4

$33.8-35$ 


$\begin{array}{lcccc}35 & 31 & 6.2 & 34.13 \pm 1.41 & 33.6-34.6 \\ 36 & 22 & 4.4 & 34.86 \pm 1.78 & 34.1-35.7 \\ 37 & 17 & 3.4 & 34.88 \pm 1.27 & 34.2-35.5 \\ 38 & 17 & 3.4 & 35.06 \pm 1.29 & 34.39-35.72 \\ 39 & 7 & 1.4 & 35.57 \pm 1.39 & 34.27-36.86 \\ 40 & 2 & 0.4 & 36.50 \pm 2.12 & 17.44-55.56\end{array}$

PT: PLACENTAL THICKNESS, CI: CONFIDENCE INTERVAL

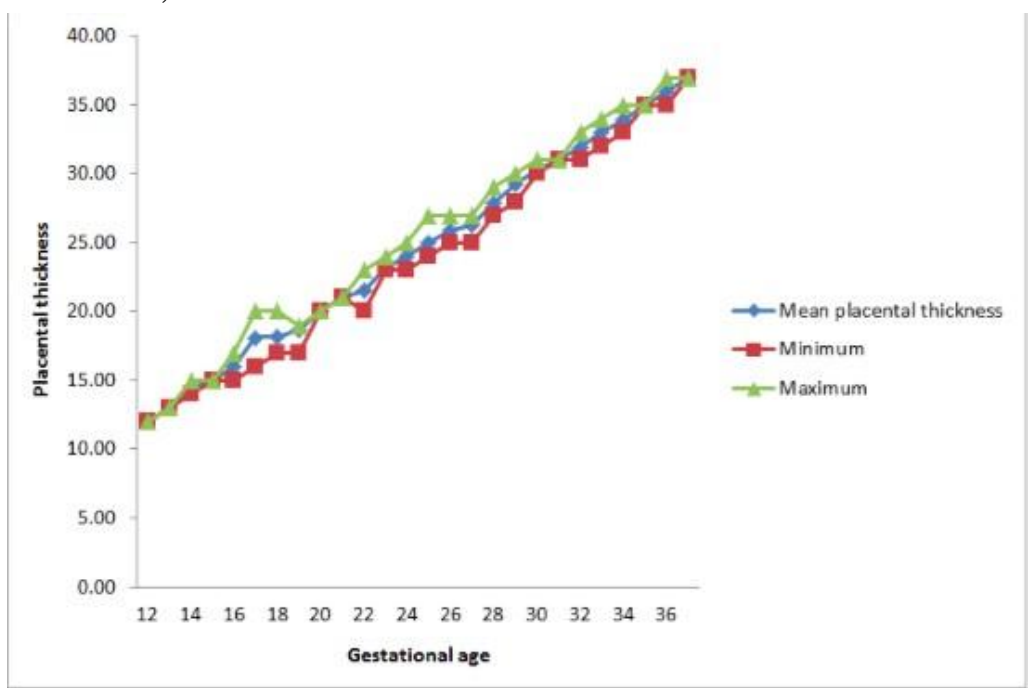

Mean values of placental thickness (in $\mathrm{mm}$ ) with $95 \%$ confidence interval vs. advancing gestational age from 11-40 weeks.
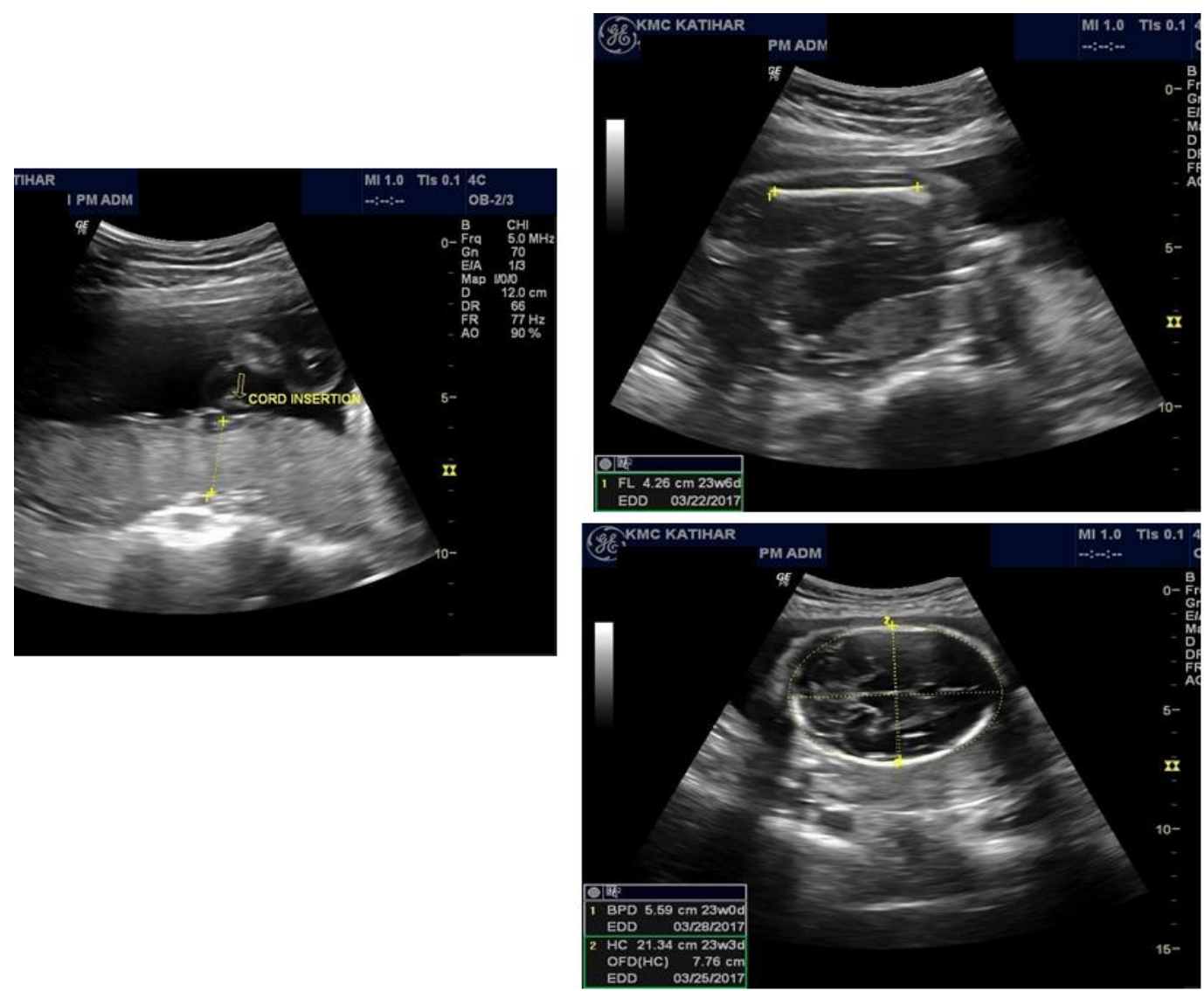

Case 1 BPD-23W 3D, HC 23W 3D, FL-23W 6D. PT-23.3MM

AVG GESTATIONAL AGE: 23 WK 3 DAYS 


\section{JMSCR Vol||07||Issue||01||Page 268-273||January}
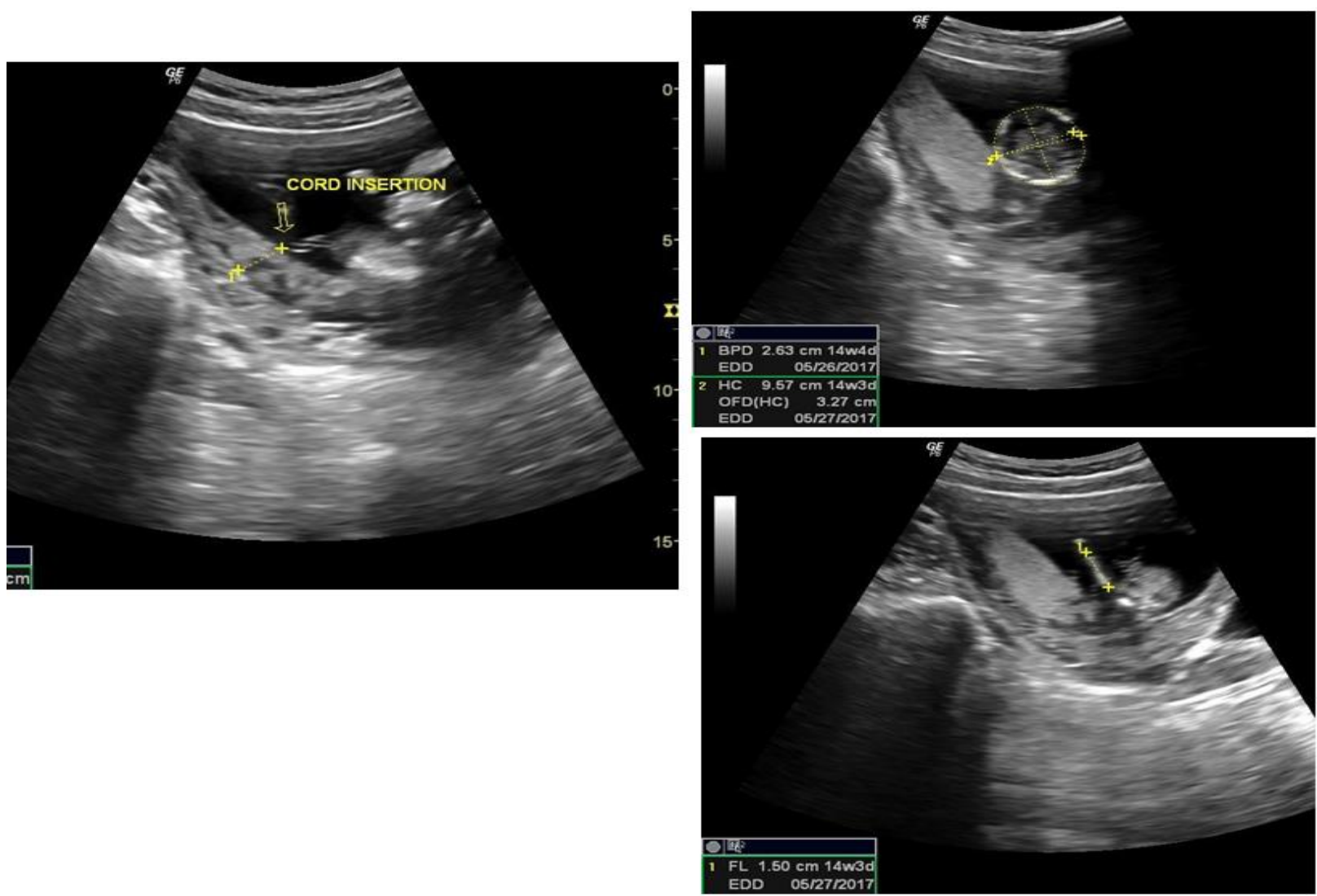

CASE 2 BPD-14W4D, HC- 14W 3D, FL - 14W 3D PT-14.3MM. AVG GESTATIONAL AGE: 14 WK 3 DAYS
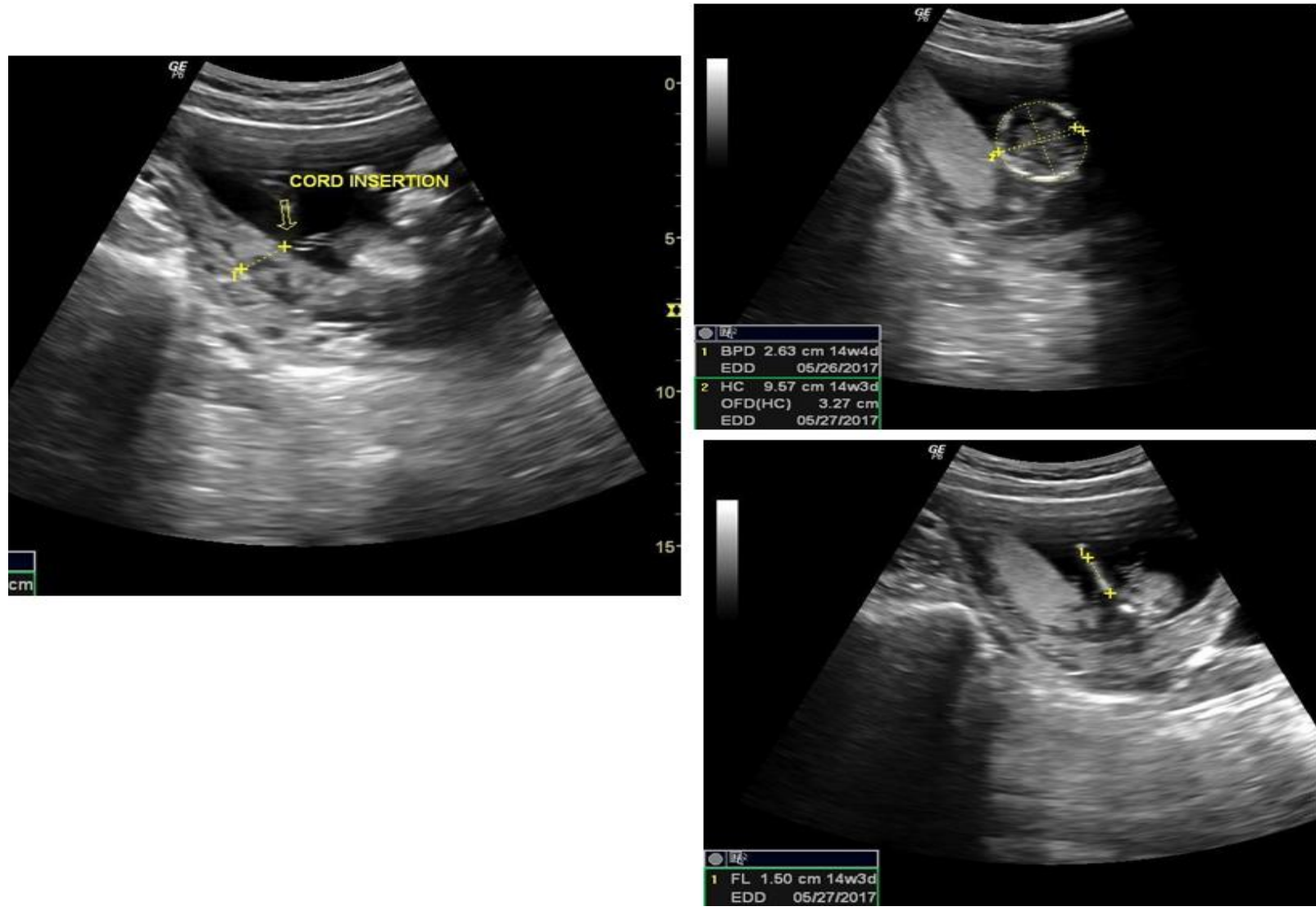

CASE 3: BPD-32W 5D, HC-33W 3D, FL-32W 6D PT-32.6MM AVG GESTATIONAL AGE32 WK 6 DAYS 


\section{Discussion}

For many years we have approached the placenta as a 'static' feature in a dynamic system. While all measurements of fetus were related to menstrual age, the placental thickness was judged as normal or abnormal based on a single "cut off" point.

The present study data confirm that placental thickness is a function of age.

Abnormal thickening or thinning must be correlated with estimates of pregnancy duration.

Hoddick et al (1985) found average placental thickness (in $\mathrm{mm}$ ) to be roughly equivalent to gestational age (in weeks). ${ }^{[5]}$

Mital $\mathrm{P}$ and Hooja $\mathrm{N}$ also found an increasing trend in the values of mean placental thickness (in $\mathrm{mm}$ ) with increase in gestational age (in weeks) and the placental thickness (in $\mathrm{mm}$ ) coincides almost exactly with the gestational age in weeks. ${ }^{[6]}$ Anupama Jain et al reported similar correlations between placental thickness and gestational age. They found placental thickness (in $\mathrm{mm}$ ) almost matched gestational age (in weeks) from 27 weeks to 33 weeks of gestation. ${ }^{\text {[7] }}$

Grannum et al reported that placental thickness would increase linearly until 33 weeks of pregnancy, after which there was gradual thinning ${ }^{[8] .}$ Other authors reported similar findings. Berkowitz et al reported gradual decrease in placental size after 32 weeks until term. ${ }^{[9]}$

Hadlock et al. suggested that one should avoid the tendency to place excessive emphasis arbitrarily on any one measurement because, in any given case, any measurement could provide the best estimate of age. ${ }^{[10]}$

The present study assessed the relationship of placenta thickness (in $\mathrm{mm}$ ) with sonographic gestational age (in weeks) and also the growth pattern with advancing gestational age. The study showed that the placental thickness (in $\mathrm{mm}$ ) increases steadily with increasing gestational age in (in weeks) in a linear fashion and almost matching the gestational age from 11-35 weeks of gestation. The rate of increase of placental thickness gradually diminished from 36-40 weeks and was less by 1-4 mm compared to gestational age (in weeks).

\section{Significance of Placental \\ Size}

Placental thickness changes are an expression of normal growth of the feto-placental unit amenable to measurement with USG and of value in describing normal physiology.

Thin placenta is often a marker for a small for dates fetuses and a sign of growth restriction. Placental thinning is also seen in patients with preeclampsia, chromosomal abnormalities and severe intrauterine infection.

Thick placentas are associated with hydrops fetalis, diabetes mellitus and intrauterine infections. Also thick placenta is associated with increased perinatal risk and increased mortality related to fetal anomalies and higher rates of both small for gestational age and large for gestational age infants at term.

\section{Conclusion}

The relationship between the placental thickness and gestational age is linear and direct.

Placental thickness (in $\mathrm{mm}$ ) measurement can be important additional parameter for estimating gestational age along with other parameters especially from 11 to 35 weeks of gestation.

The mean placental thickness were also correlated with gestational ages between 11-35 weeks with linear regression model.

Placental thickness increases with increasing gestational age and almost matches it from 11 to 35 weeks of gestation.

The relationship of placental thickness with gestational age falls marginally and the rate of growth of placental thickness decreases after 36 weeks of gestation and is less than gestational age by $1-3 \mathrm{~mm}$.

\section{Reference}

1. Heera Tongsong, Pongrak Boonyanurak. Placental thickness in the first half of 
pregnancy. J Clin Ultrasound 2004; 32:5: 231-234

2. Jauniaux E. Placental ultrasonographic measurements:

a. What can we learn and is it worth doing routinely Ultrasound Obstet. Gynaecol. 1992; 2:241-242

3. Ghosh A, Tang MH, Lam YH et al. Ultrasound measurement of the placental thickness to detect pregnancies affected by homozygous alpha thalassaemia-1. Lancet 1994; 344: 988-989

4. Nyberg DA, Finberg HJ. The placenta, placental membranes and umbilical cord. Journal on diagnostic ultrasound of fetal anomalies. 1990;21(4):623-75.

5. Hoddick WK, Mahoney BS, Callen FW, Filly RA.

a. Placental thickness. J Ultrasound Med. 1985; 4:479-482.

6. Mittal P, Hooja N, Mehndiratta. Placental thickness- a sonographic parameter for estimating gestational age of the fetus. Ind J Radiol Imag. 2002; 12:4: 553- 554

7. Jain A, Kumar G, Agarwal U, Kharakwal S. Pacental thickness - a sonographic indicator of gestational age. Journal of Obstret. and Gynaecol. of India. 2001;51:48-49

8. Granum PAT, Hobbins JC. The placenta. Radiology Clinic North America. 1982; 20:353.

9. Granum PAT, Berkowitz RL, Hobbins JC. The ultrasonic changes in the maturing placenta and their relation of fetal pulmonic maturity. Am J Obstret. and Gynecol. 1979; 133:915-922.

10. Hadlock FP, Harrist RB, Martinez-Poyer J. How accurate is second trimester fetal dating? J Ultrasound Med. 1991;10:55761. 